\title{
DEUTERIUM CHEMISTRY IN THE PRIMORDIAL GAS
}

\author{
Daniele Galli and Francesco Palla \\ Osservatorio Astrofisico di Arcetri \\ Largo Enrico Fermi 5 \\ I-50125 Firenze, Italy \\ galli,palla@arcetri.astro.it
}

\begin{abstract}
We review and update some aspects of deuterium chemistry in the postrecombination Universe with particular emphasis on the formation and destruction of HD. We examine in detail the available theoretical and experimental data for the leading reactions of deuterium chemistry and we highlight the areas where improvements in the determination of rate coefficients are necessary to reduce the remaining uncertainties. We discuss the cooling properties of HD and the modifications to the standard cooling function introduced by the presence of the cosmological radiation field. Finally, we consider the effects of deuterium chemistry on the dynamical collapse of primordial clouds in a simple "top-hat" scenario, and we speculate on the minimum mass a cloud must have in order to be able to cool in a Hubble time.
\end{abstract}

Subject headings: atomic and molecular processes - early Universe

\section{Introduction}

The formation of $\mathrm{H}_{2}$ and HD molecules in the post-recombination Universe plays a central role in the evolution of gas condensations. Even trace abundances of these molecules strongly affect the cooling properties of the primordial gas which would be otherwise an extremely poor radiator (cooling by Ly- $\alpha$ photons is ineffective at temperatures less than $\sim 10^{4} \mathrm{~K}$ ).

The chemistry of the early Universe has been investigated in several studies starting with the seminal paper by Lepp \& Shull (1983). We mention in particular the work of Puy et al. (1993), Galli \& Palla (1998; hereafter GP) and the comprehensive analysis of deuterium chemistry by Stancil, Lepp \& Dalgarno (1998; hereafter SLD). The abundances of $\mathrm{H}_{2}$ and HD predicted at low redshift in these studies are of the order of $10^{-6}$ and $10^{-9}$, respectively, depending on the cosmological model adopted (see Palla, Galli \& Silk 1995 for the variation of the chemical abundances with the assumed baryon-to-photon ratio). A comparison of the abundance of HD obtained at $z=10$ by GP $\left(n[\mathrm{HD}] / n[\mathrm{H}]=1.1 \times 10^{-9}\right)$, and $\mathrm{SLD}\left(n[\mathrm{HD}] / n[\mathrm{H}]=1.6 \times 10^{-9}\right)$ for the same cosmological model ( $h=0.5, \Omega_{0}=1, \Omega_{b}=0.0367$ ) shows satisfactory agreement, although this might not be completely significant since both calculations were based on a compilation of reaction rates obtained 
basically from the same sources. To repeat the words of SLD, "further studies are needed to reduce the uncertainty in the HD abundance". One of the goals of this paper is to review the progress made in our understanding of HD chemistry in the three years subsequent to the publication of these studies. We will outline the improvements which have occurred in the meantime, and discuss the remaining uncertainties.

From an observational standpoint, the abundance of atomic deuterium and molecular hydrogen has been measured in several cosmological clouds at redshift $z \simeq 2-3$ in absorption along the line of sight to bright quasars. Deuterium in particular has attracted renewed attention because of the controversy about its abundance in high-redshift Ly- $\alpha$ clouds (see Hogan 1998 for an account and a resolution of the controversy). Molecular hydrogen at high redshift was first detected by Levshakov \& Varshalovich (1985) in a damped Ly- $\alpha$ system at $z=2.8$. Since then, the presence of $\mathrm{H}_{2}$ has

been confirmed in at least four additional systems (see e.g. Levshakov et al. 2000 and references therein).

In Fig. 1 we compare observational data on $\mathrm{D}$ and $\mathrm{H}_{2}$ at high redshift with the corresponding abundances calculated with the standard model of GP that follows the homogenous expansion of the universe. The agreement between the theoretical and observed deuterium abundance is excellent. In the case of $\mathrm{H}_{2}$, one should keep in mind the sensitivity of this molecule to ambient conditions in Ly- $\alpha$ clouds, such as the stronger ultraviolet radiation field and the lower dust content with respect to local interstellar medium. These fctors can account for the considerable spread of observed abundances. Despite the complex phenomenology associated with damped Ly- $\alpha$ systems and the resulting uncertainty in the interpretation of the results, it is encouraging, to say the least, to witness the emergence of the observational foundations of the highly theoretical discipline of primordial chemistry.

\section{Chemical Reactions}

The formation of HD in the primordial gas follows two main routes, involving a deuteron exchange with $\mathrm{H}_{2}$ :

$$
\mathrm{D}+\mathrm{H}_{2} \rightarrow \mathrm{HD}+\mathrm{H}
$$

and

$$
\mathrm{D}^{+}+\mathrm{H}_{2} \rightarrow \mathrm{HD}+\mathrm{H}^{+}
$$

Being an isotopic modification of the the most fundamental three-electron interaction, namely, the $\mathrm{H}+\mathrm{H}_{2}$ reaction, reaction (1) has received considerable interest. Thermal rate constants for this reaction have been measured by Ridley, Schulz, \& LeRoy (1966), Westenberg \& de Haas (1967), Mitchell \& LeRoy (1973), and Michael \& Fisher (1990) over a wide range of temperatures. Theoretical calculations employing statistical, semiclassical and quantal method have been performed by several groups, and show very good agreement with each other and with the experimental data. 
The most recent studies are by Zhang \& Miller (1989), Michael, Fisher, \& Bowman (1990), Mielke et al. (1994), and Charutz, Last, \& Baer (1997). These results are compared in the usual Arrhenius plot shown in Fig. 2 (the high-temperature experimental results of Michael \& Fisher 1990 are not shown). In this paper we adopt the reaction rate computed by Mielke et al. (1994) with the DMBE surface (Varandas et al. 1987) which predicts much more accurate low-temperature kinetics than other surfaces. Their results agree with the values computed by Michael \& Fisher (1990) (with the same surface) within $\sim 20 \%$. We also note that Zhang \& Miller (1989) computed state-by-state cross sections for $v J \rightarrow v^{\prime} J^{\prime}$ transitions and state-to-state rate constants in the temperature range 200-1000 K. The agreement with the experimental cross section values at $E \simeq 1 \mathrm{eV}$ (Phillips, Levene, \& Valentini 1989) is within a factor $\sim 2$.

Reaction (2) represents the major source of HD in diffuse interstellar clouds (Dalgarno, Weisheit, \& Black 1973). Its rate coefficient is almost constant and close to the Langevin value $(2 \times$ $10^{-9} \mathrm{~cm}^{3} \mathrm{~s}^{-1}$, see Fig. 3). The reaction rate has been measured in the laboratory by Fehsenfeld et al. (1973, at $T=200$ and $278 \mathrm{~K}$ ) using a flowing afterglow technique, and by Henchman, Adams, \& Smith (1981, at $T=205$ and $295 \mathrm{~K}$ ) using a variable-temperature selected ion flow tube technique (see also Smith, Adams, \& Alge 1982). Gerlich (1982) performed accurate quantum-mechanical calculations of the rate coefficient, which are in excellent agreement with the experimental results of Henchmann et al. (1981) at $T=295 \mathrm{~K}$, but less at $T=205 \mathrm{~K}$ (still within a factor $\sim 2$ ). There is a discrepancy with the results of Fehsenfeld et al. (1973) at both temperatures.

The destruction of HD occurs mainly via the reverse reactions of (1) and (2),

$$
\mathrm{HD}+\mathrm{H} \rightarrow \mathrm{D}+\mathrm{H}_{2}
$$

and

$$
\mathrm{HD}+\mathrm{H}^{+} \rightarrow \mathrm{D}^{+}+\mathrm{H}_{2}
$$

In general, the rate coefficients for the forwards and reverse chemical reactions are related by the standard thermodynamic expression (e.g. Berry, Rice \& Ross 1980)

$$
\ln \left(\frac{k_{\mathrm{f}}}{k_{\mathrm{r}}}\right)=-\frac{\Delta H^{0}}{R T}+\frac{\Delta S^{0}}{R}
$$

where $R$ is the universal gas constant, and $\Delta H^{0}, \Delta S^{0}$ are the enthalpy and entropy changes. Thus, if the rate $k_{\mathrm{f}}$ is knwon, the rate for the reverse reaction $k_{\mathrm{r}}$ can be obtained directly from eq. (5). From the differences in the zero point vibrational energies of $\mathrm{H}_{2}$ and $\mathrm{HD}$ and in the ionization potentials of $\mathrm{H}$ and $\mathrm{D}$, one obtains the enthalpy changes for reactions (1) and (2): $\Delta H^{0} / R=-412 \mathrm{~K}$ and $-462 \mathrm{~K}$, respectively. The entropy change $\Delta S^{0} / R$ can be calculated on statistical grounds (see e.g. Flower 2000) and is the same for both reactions: $\Delta S^{0} / R=\ln 2$ for para- $\mathrm{H}_{2}, \Delta S^{0} / R=\ln (2 / 3)$ for ortho- $\mathrm{H}_{2}$. These values are for reactant and product molecules in their ground states. Both the entropy and the enthalpy changes are modified when rovibrationally excited molecular states are involved (Flower 2000). 
Rather than using eq. (5), in this paper we prefer to provide independent fitting formulae for both the direct and reverse reactions that dominate the chemistry of deuterium in the primordial gas. For specific applications, the reader may adopt the reaction rate of the forward (or reverse, if better constrained) reaction, and compute the rate of the reverse (forward) reaction from the thermodynamic relation expressed by eq. (5).

The rate coefficient of reaction (3) has been calculated by Shavitt (1959) using a semiempirical $\mathrm{H}_{3}$ energy surface. Only sparse laboratory data exist for this reaction. At $T=10^{3} \mathrm{~K}$, the experimental result of Boato et al. (1956) is within $\sim 20 \%$ from the value calculated by Shavitt (1959). At lower temperatures $(720-880 \mathrm{~K})$, the theoretical rate is a factor $\sim 2$ lower than the experimental data by van Meersche (1951), but the extrapolation of the adopted $\mathrm{H}_{3}$ energy surface introduces significant uncertainty in the results at low temperatures (see Fig. 4). As a challenge to chemical physicists, we recall the words of Shavitt (1959): "it is unfortunate that for a reaction as basically important as the one considered here, the experimental data are so incomplete and inconclusive".

Since reaction (4) is endothermic by $0.0398 \mathrm{eV}(462 \mathrm{~K})$, the removal of $\mathrm{HD}$ at low temperatures is reduced by a factor $\exp (-462 / T)$, and this can lead to significant enhancement of the $\mathrm{HD} / \mathrm{H}_{2}$ ratio (fractionation). The rate coefficient of this reaction has been measured in the laboratory by Henchmann et al. (1981) at $T=205$ and $295 \mathrm{~K}$, and the results are in good agreement with the values obtained by applying the principle of detailed balance to the reverse reaction (2). As in GP, we adopt the rate coefficient calculated by Gerlich (1982) over the temperature range 30-600 K (see Fig. 5).

Finally, the relative abundance of $\mathrm{D}$ and $\mathrm{D}^{+}$is determined by the charge exchange reactions

$$
\mathrm{H}^{+}+\mathrm{D} \rightarrow \mathrm{H}+\mathrm{D}^{+}
$$

and its reverse

$$
\mathrm{H}+\mathrm{D}^{+} \rightarrow \mathrm{H}^{+}+\mathrm{D}
$$

The cross section for reaction (6) was computed by Matveenko (1974) and Hunter \& Kuriyan (1977) for energies from $10^{-3}$ to $7.5 \mathrm{eV}$. The two results differ by a factor $\sim 2$ at low energies for reasons unclear. Subsequent calculations by Hodges \& Breig (1993), and, more recently, by Igarashi \& Lin (1999) and Zhao, Igarashi, \& Lin (2000), confirm the validity of the results of Hunter \& Kuriyan (1977), and improve significantly the accuracy of the calculations around $\sim 10^{-3} \mathrm{eV}$. Good agreement was also found with the experimental measurements of Newman et al. (1982) and Esry et al. (2000). The cross section of reaction (7) was also calculated by Igarashi \& Lin (1999) and Zhao et al. (2000). Unfortunately, no experimental data are available for this reaction.

Watson (1976) estimated the rate of reactions (6) and (7) and Watson, Christensen \& Deissler (1978) calculated the rate coefficient on the basis of the cross section obtained by Hunter \& Kuriyan (1977). These rates have been widely adopted in studies of deuterium chemistry. Since reaction (6) has a threshold of $43 \mathrm{~K}$, the rate coefficient for reaction (7) is usually obtained by multiplying that of reaction (6) by $\exp (43 / T)$. The situation has been reanalyzed recently by Wolf Savin (2001) who 
computed accurate rates for reactions (6) and (7) from the cross sections of Igarashi \& Lin (1999)

and Zhao et al. (2000). These results are compared to those of Watson et al. (1978) in Figs. 6 and 7 .

In Table 1 we collect accurate fitting formulae (computed by us or by the authors quoted in the references) for the chemical reactions discussed in this section. These expressions update and replace the corresponding formulae given in Table 2 of GP.

In addition to these reactions, additional contributions to the formation of HD in the early Universe come from the associative detachment reactions

$$
\mathrm{D}+\mathrm{H}^{-} \rightarrow \mathrm{HD}+e
$$

and

$$
\mathrm{D}^{-}+\mathrm{H} \rightarrow \mathrm{HD}+e,
$$

whose rate coefficients however are not known, and can only be estimated from the corresponding $\mathrm{H}$ reactions (see SLD). Finally, minor contributions to the formation of $\mathrm{HD}$ come from the radiative association reaction

$$
\mathrm{H}+\mathrm{D} \rightarrow \mathrm{HD}+h \nu
$$

whose rate coefficient was computed by Stancil \& Dalgarno (1997), and from reactions involving less abundant deuterated species like $\mathrm{HD}^{+}$and $\mathrm{H}_{2} \mathrm{D}^{+}$(see SLD for details).

Generally, the chemistry of HD in the primordial gas is dominated by the ion-neutral reactions (2) and (4) in a gas of low density (e.g. the primordial gas before the formation of the first structures), whereas the neutral-neutral reactions (1) and (3) become more important in conditions of high density and temperature (cloud collapse, shocked gas).

\section{Heating and Cooling}

In order to calculate the cooling properties of $\mathrm{HD}$, one should know the population of all rovibrational levels. In steady-state, these are obtained by solving the balance equations

$$
x_{J} \sum_{J^{\prime}}\left[R_{J J^{\prime}}\left(T_{\mathrm{rad}}\right)+C_{J^{\prime}}\left(n, T_{\mathrm{gas}}\right)\right]=\sum_{J^{\prime}} x_{J^{\prime}}\left[R_{J^{\prime} J}\left(T_{\mathrm{rad}}\right)+C_{J^{\prime} J}\left(n, T_{\mathrm{gas}}\right)\right],
$$

where $J$ and $J^{\prime}$ indicate a generic couple of rovibrational levels. The collisional transition probabilities $C_{J J^{\prime}}\left(n, T_{\text {gas }}\right)$ and $C_{J^{\prime} J}\left(n, T_{\text {gas }}\right)$ are obtained by multiplying the corresponding excitation coefficients, $\gamma_{J J^{\prime}}\left(T_{\text {gas }}\right)$ and $\gamma_{J^{\prime} J}\left(T_{\text {gas }}\right)$, and the density of the colliding species. The terms $R_{J J^{\prime}}$ and $R_{J^{\prime} J}$ are the radiative excitation and de-excitation rates, that can be expressed in terms of the Einstein coefficients $A_{J J^{\prime}}$ and $B_{J J^{\prime}}$,

$$
R_{J J^{\prime}}= \begin{cases}A_{J J^{\prime}}+B_{J J^{\prime}} u\left(\nu_{J J^{\prime}}, T_{\mathrm{rad}}\right), & J^{\prime}<J, \\ B_{J J^{\prime}} u\left(\nu_{J J^{\prime}}, T_{\mathrm{rad}}\right), & J^{\prime}>J\end{cases}
$$


where $u\left(\nu_{J J^{\prime}}, T_{\mathrm{rad}}\right)$ is the energy density of the cosmic background radiation $(\mathrm{CBR})$ per unit frequency at the temperature $T_{\text {rad }}$,

$$
u\left(\nu_{J J^{\prime}}, T_{\mathrm{rad}}\right)=\frac{8 \pi h \nu_{J J^{\prime}}^{3}}{c^{2}}\left[\exp \left(h \nu_{J J^{\prime}} / k T_{\mathrm{rad}}\right)-1\right]^{-1} .
$$

In the primordial gas, collisional excitation of $\mathrm{HD}$ is dominated by collisions with $\mathrm{H}$, and, to a less extent, with He. The coefficients for inelastic scattering of He-HD were computed by Green (1974) and Schaefer (1990) at temperatures $T \leq 600 \mathrm{~K}$, for $0 \leq J \leq 3$ and $\Delta J=+1,+2$. Collisional coefficients for rotational excitation of the system H-HD were usually derived by scaling the He-HD values with the square root of the ratio of the reduced masses of the two systems, $\gamma_{\mathrm{H}-\mathrm{HD}}=\left(\mu_{\mathrm{He}-\mathrm{HD}} / \mu_{\mathrm{H}-\mathrm{HD}}\right)^{1 / 2} \gamma_{\mathrm{He}-\mathrm{HD}}$, where $\left(\mu_{\mathrm{He}-\mathrm{HD}} / \mu_{\mathrm{H}-\mathrm{HD}}\right)^{1 / 2}=1.51$ (see e.g. Timmerman 1996, GP). In the words of Timmermann (1996), "this assumption is however an educated guess, and data on $H-H D$ are urgently needed". These data were recently provided by Flower \& Roueff (1999) (for $\mathrm{H}$ and $\mathrm{H}_{2}$ ) and Roueff \& Zeippen (1999) (for He), using full quantum-mechanical methods and updated energy potential surfaces to evaluate rovibrational excitation coefficients for collisions of $\mathrm{HD}$ with $\mathrm{H}, \mathrm{H}_{2}$ and $\mathrm{He}$. The results for $\mathrm{H}-\mathrm{HD}$ collisions computed by Flower \& Roueff (1999) in the temperature range $100 \leq T_{\text {gas }} \leq 2000 \mathrm{~K}$ for $v \leq 2$ and $J \leq 9$ for a total of 30 rovibrational levels are available from the CCP7 server http://ccp7.dur.ac.uk/.

The energy levels and the Einstein coefficients $A_{J J^{\prime}}$ of HD were calculated by Abgrall et al. (1982) who considered both dipole and quadrupole transitions and included a large number of rovibrational levels. Since the energy spacing of the rotational levels of HD is quite large, $E_{1} / k=128 \mathrm{~K}, E_{2} / k=383 \mathrm{~K}, E_{3} / k=764 \mathrm{~K}$, etc., GP computed the HD cooling function with a simple four-level system $(J=0-3)$ adopting the collisional coefficients of Schaefer (1990). Flower et al. (2000) updated the calculations of GP adopting the collisional rate coefficients of Flower \& Roueff (1999) and Roueff \& Zeippen (1999). The HD cooling function computed by Flower et al. (2000) is also available from the CCP7 server. A useful approximation in the low-density limit is the expression

$$
\Lambda_{\mathrm{HD}}[n(\mathrm{H} \rightarrow 0)]=2 \gamma_{10}\left(T_{\text {gas }}\right) E_{10} e^{-E_{10} / k T_{\text {gas }}}+(5 / 3) \gamma_{21}\left(T_{\text {gas }}\right) E_{21} e^{-E_{21} / k T_{\text {gas }}},
$$

where $E_{10} / k=128 \mathrm{~K}, E_{21} / k=255 \mathrm{~K}$ and the collisional rate coefficients $\gamma_{J J^{\prime}}$ are given by Flower \& Roueff (1999):

$$
\gamma_{10}\left(T_{\text {gas }}\right)=4.4 \times 10^{-12}+3.6 \times 10^{-13}\left(\log T_{\text {gas }}\right)^{0.77} \mathrm{~cm}^{3} \mathrm{~s}^{-1}
$$

and

$$
\gamma_{21}\left(T_{\text {gas }}\right)=4.1 \times 10^{-12}+2.0 \times 10^{-13}\left(\log T_{\text {gas }}\right)^{0.92} \mathrm{~cm}^{3} \mathrm{~s}^{-1}
$$

The comparison between the HD cooling rate calculated by GP and Flower et al. (2000), shown in Fig. 8 is instructive. A simple four-level molecule is able to predict quite accurately the cooling rate in a wide range of temperatures $\left(T_{\text {gas }} \lesssim 2000 \mathrm{~K}\right)$ and densities $\left(n[\mathrm{H}] \lesssim 10^{7} \mathrm{~cm}^{-3}\right)$, but of course 
fails badly in the high-temperature, high-density regime. However, for cosmological applications, it is important to keep in mind that the HD cooling function of Flower et al. (2000) has been computed assuming that the temperature of the CBR is much smaller than the gas temperature. This approximation is valid at low redshifts (where the two temperatures differ by more than a factor $\sim 10$ for $z \lesssim 20$ ), but becomes increasingly inaccurate at higher redshifts. Since most cosmological scenarios of structure formation begin at $z \simeq 100$, when $T_{\text {rad }} \simeq 300 \mathrm{~K}$, the level population of molecules is strongly affected by stimulated emission and absorption. In such conditions, molecules may become an effective heating source for the gas, because the rate of collisional de-excitation of the rovibrational levels is faster than their radiative decay (Khersonskii 1986; Puy et al. 1993).

As an illustration of this effect, we plot in Fig. 9 the net heat transfer function $(\Gamma-\Lambda)_{\mathrm{HD}}$ computed with the GP model for $n(\mathrm{H})=1 \mathrm{~cm}^{-3}$ as function of gas temperature at three selected redshifts. Note that for $T_{\text {gas }}>T_{\text {rad }}$ the cooling function is significantly decreased from the value computed with $T_{\text {rad }}=0$, because of the radiative depopulation of excited states. The sudden drop signals the condition $T_{\text {gas }}=T_{\text {rad }}$. Finally, for $T_{\text {gas }}<T_{\text {rad }}$ the function changes sign and becomes a net heating term for the gas. In cosmological simulations the heat transfer should be computed self-consistently at each redshift.

\section{Application: cloud collapse at $z \simeq 100$}

In both cold dark matter and baryonic dark matter cosmological scenarios, the first objects predicted to enter in the nonlinear stage are the smallest ones. In cold dark matter models, it is expected that overdense regions with masses $10^{5}-10^{7} M_{\odot}$ first collapse in the redshift range $10 \lesssim$ $z \lesssim 100$ (see e.g. Cen, Ostriker, \& Peebles 1993). The crucial question is whether molecular cooling allows the baryonic component to dissipate its kinetic energy and collapse on a timescale shorter than a Hubble time. This important question is fully addressed e.g. in the 1-D hydrodynamical calculations by Haiman, Thoul, \& Loeb (1996) and in the 3-D numerical simulations presented by Abel, Anninos, \& Norman (1997) and Bromm, Coppi, \& Larson (1999). Here, we consider a particular issue related to the choice of the initial conditions for collapse calculations.

Following Tegmark et al. (1997), we consider the growth of a "top-hat" overdensity region, an isolated spherical perturbation in a uniform density Universe (see e.g. Padmanabhan 1993). The radius of this region increases at a slower rate than the scale factor of the Universe (but still obeys the Friedman's equations) and, after reaching a maximum value (turnaround), recollapses to a point. The formation of a singularity is clearly an artifact of the simplified assumptions of the "top-hat" model. In a realistic situation, gas dynamical process (internal pressure, shocks) will eventually halt the collapse of the baryonic component at some finite value of the density. The resulting quasi-equilibrium configuration is a virialized "halo", and its subsequent evolution depends on the ability of the gas to cool on a timescale shorter than a dynamical timescale.

We show in Fig. 10 the results of a sample run of the Tegmark et al. (1997) evolutionary 
model, obtained with our chemical network and molecular cooling prescriptions. For the case shown, the cloud reaches virialization at $z_{\mathrm{vir}}=110$, where the temperature is $T_{\text {vir }}=2000 \mathrm{~K}$. After virialization, the gas density is assumed to remain constant and uniform for the rest of the run, and we follow the cooling of the gas due to $\mathrm{H}_{2}$ and $\mathrm{HD}$ molecules. This is clearly a poor approximation since the cooling of the gas will of course induce an increase in the the density, and the postvirialization evolution of the cloud must be followed with a full hydrodynamical calculation (Galli et al., in preparation). Nevertheless, it is instructive to consider how rapidly the baryons are able to dissipate the thermal energy of the cloud via molecular cooling. As we see in Fig. 10, recombination reduces the ionization fraction of the cloud to negligible values, weakening the Compton cooling of the gas (since $T_{\text {rad }}<T_{\text {gas }}$ ). The rapid rise of $\mathrm{H}_{2}$ (via the $\mathrm{H}^{-}$channel) around $z_{\text {vir }}$ causes the sudden cooling of the gas from $T_{\text {vir }}$ down to a few hundred degrees, and the subsequent increase in the HD abundance further reduces the gas temperature down to few tens of degrees, establishing again thermal coupling of gas and cosmic radiation at redshift $z \simeq 10$.

Loosely speaking, a Hubble time corresponds to the redshift dropping by a factor $2^{2 / 3} \simeq$ 1.6. For the particular case considered in this illustrative example, at a resdhift $z=z_{\text {vir }} / 1.6 \simeq$ 70 the cloud temperature has dropped to $T_{\text {gas }} \simeq 350$, i.e. of a factor $\sim 6$ with respect to the virial temperature. Molecular cooling (mostly $\mathrm{H}_{2}$ in this range of redshift) therefore enables the cloud to cool significantly within a Hubble time, and eventually to collapse and form luminous objects. Cooling by HD appears to induce a further substantial reduction of the temperature at later times, but the subsequent evolution of the cloud can only be followed with a realistic calculation. However, it must be kept in mind that the mass scale of the fragments formed by the collapse of the cloud depends sensitively on the gas temperature $\left(M_{J} \sim \rho^{-1 / 2} T_{\text {gas }}^{3 / 2}\right)$. The cooling induced by the formation of HD may in fact reduce the gas temperature below $\sim 100 \mathrm{~K}$ and therefore enable the formation of primordial low-mass stars or even brown dwarfs, a possibility recently explored by Uheara \& Inutsuka (2000) (see also Flower \& Pineau des Forêts 2001). These exciting results represent a radical cange of perspective in the field of primordial star formation and deserve further investigation.

\section{Conclusions}

We have examined the most effective chemical reactions leading to the formation/destruction of HD molecules in the early Universe, and presented a list of accurate reaction rate coefficients for primordial chemistry calculations that update those adopted in previous studies. We have analyzed the heating/cooling properties of HD molecules, stressing the relevance of a self-consistent calculation of the energy transfer rate between gas and radiation for cosmological applications. As an illustration, we have presented a simplified model for the evolution of density perturbations in the expanding Universe, following previous investigations by Haiman et al. (1996) and Tegmark et al. (1997), but including our comprehensive and updated chemical network. These results, together with recent findings by Uheara \& Inutsuka (2000) and Flower \& Pineau des Forêts (2001) 
underline the substantial contribution of HD to gas cooling during the collapse of primordial clouds. A preliminary conclusion from these studies is that $\mathrm{HD}$ is at least as important as $\mathrm{H}_{2}$ in determining the thermal balance of zero-metal clouds.

This work is supported by the Italian Ministry for the University and for Scientific and Tech-

nological Research (MURST) through a COFIN-2000 grant. It is a pleasure to thank Guillaume Pineau des Forêts and Malcolm Walmsley for informative dicussions on deuterium chemistry. We also thank A. Dalgarno, S. N. Shore and an anonymous referee for useful comments on an earlier version of this paper.

\section{REFERENCES}

Abel, T., Anninos, P., Norman, M. 1997, New Astr., 2181

Abgrall, H., Roueff, E., \& Viala, Y. 1982, A\&AS, 50, 505

Berry, R. S., Rice, S. A., \& Ross, J., 1980, Physical Chemistry (New York: Wiley), p. 756

Boato, A., Careri, G., Cimino, S., Molinari, S., \& Volpi, C. 1956, J. Chem. Phys., 24, 783

Bromm, V., Coppi, P. S., \& Larson, R. B. 1999, ApJ, 527, L5

Burles, S., \& Tytler, D. 1998a, ApJ, 499, 699

Burles, S., \& Tytler, D. 1998b, ApJ, 507, 732

Cen, R., Ostriker, J. P., \& Peebles, P. J. E. 1993, ApJ, 415, 423

Charutz, D. M., Last, I., \& Baer, M. 1997, J. Chem. Phys. 106, 7654

Dalgarno, A., Weisheit, J. C., \& Black, J. H. 1973, Astrophys. Lett., 14, 77

D’Odorico, S., Dessauges-Zavadsky, M., \& Molaro, P. 2001, A\&A, 368, L21

Esry, B. D., Sadeghpour, H. R., Wells, E., \& Ben-Itzhak, I. 2000, J. Phys. B, 33, 5329

Fehsenfeld, F. C., Dunkin, D. B., Ferguson, E. E., \& Albritton, D. L. 1973, ApJ, 183, L25

Fehsenfeld, F. C., Albritton, D. L., Bush, Y. A., Fournier, P. G., Govers, T. R., \& Fournier, J. 1974, J. Chem. Phys., 61, 2150

Flower, D. R., \& Roueff, E. 1999, MNRAS, 309, 833

Flower, D. R. 2000, MNRAS, 318, 875

Flower, D. R., Le Bourlot, J., Pineau des Forêts, G., \& Roueff, E. 2000, MNRAS, 314, 753 
Flower, D. R., \& Pineau des Fôrets, G. 2001, MNRAS, 323, 627

Galli, D., \& Palla, F. 1998, A\&A, 456, 631 (GP)

Gerlich, D., 1982, in Symp. on Atomic and Surface Physics, W. Lindinger, F. Howorka, T. D. Märk, F. Egger eds. (Dordrecht: Kluwer), p. 304

Green, S. 1974, Physica, 76, 609

Haiman, Z., Thoul, A. A., \& Loeb, A. 1996, ApJ, 464, 523

Henchman, M. J., Adams, N. G., \& Smith, D. 1981, J. Chem. Phys., 75, 1201

Hodges, R. R., Jr., \& Breig, E. L. 1993, J. Geophys. Res., 98, 1581

Hogan, C. J. 1998, in Primordial Nuclei and their Galactic evolution, N. Prantzos, M. Tosi, \& R. von Steiger eds. (Dordrecht: Kluwer), p. 127

Hunter, G., \& Kuriyan, M. 1977, Proc. R. Soc. London Ser. A, 358, 321

Igarashi, A., \& Lin, C. D. 1999, Phys. Rev. Lett., 83, 4041

Khersonskii, V. K. 1986, Astrophysics, 24, 114

Lepp, S. \& Shull, J. M. 1983, ApJ, 270, 578

Lepp, S. \& Shull, J. M. 1984, ApJ, 280, 465

Levshakov, S. A., \& Varshalovich, D. A. 1985, MNRAS, 212, 517

Levshakov, S. A., Molaro, P., Centurión, M., D’Odorico, S., Bonifacio, P., \& Vladilo, G. 2000, A\&A, 361, 803

Matveenko, A. V. 1974, Sov. Phys. JETP, 38, 1082

Michael, J. V., \& Fisher, J. R. 1990, J. Phys. Chem., 93, 3318

Michael, J. V., \& Fisher, J. R., \& Bowman, Q. S. 1990, Science, 249, 269

Mielke, S. L., Lynch, G. C., Thrular, D. G., \& Schwenke, D. W. 1994, J. Phys. Chem., 98, 8000

Mitchell, D. N., \& LeRoy, D. J. 1973, J. Chem. Phys., 58, 3449

Newman, J. H., Cogan, J. D., Ziegler, D. L., Nitz, D. E., Rundel, R. D., Smith, K. A., \& Stebbings, R. F. 1982, Phys. Rev. A, 25, 2976

O’Meara, J. M., Tytler, D., Kirkman, D., Suzuki, N., Prochaska, J. X., Lubin, D., \& Wolfe, A. M. 2001, ApJ, 522, 718

Padmanabhan, T. 1993, Structure formation in the Universe, Cambridge Univ. Press 
Palla, F., Galli, D., \& Silk, J. 1995, ApJ, 451, 44

Phillips, D. L., Levene, H. B., \& Valentini, J. J. 1989, J. Chem. Phys. 90, 1600

Puy, D., Alecian, G., Le Bourlot, J., Léorat, J., \& Pineau des Fôrets, G. 1993, A\&A, 267, 337

Ridley, B.A., Schulz, W.R., LeRoy, D.J. 1966, J. Chem. Phys., 44, 3344

Roueff, E., \& Zeippen, C. J. 1999, A\&A, 343, 1005

Rozhenstein, V. B., Gershenzon, Yu. M., Ivanov, A. V., Il'in, S. D., Kurcheryavii, S. I., \& Umanskii, S. Ya. 1985, Chem. Phys. Lett., 121, 89

Schaefer, J. 1990, A\&AS, 85, 1101

Schofield, K. 1967, Planet. Space Sci., 15, 643

Shavitt, I. 1959, J. Chem. Phys., 31, 1359

Smith, D., Adams, N. G., \& Alge, E. 1982, ApJ, 263, 123

Stancil, P. C., \& Dalgarno, A. 1997, ApJ, 490, 76

Stancil, P. C., Lepp, S., \& Dalgarno, A. 1998, ApJ, 509, 1 (SLD)

Tegmark, M., Silk, J., Rees, M. J., Blanchard, A., Abel, T., \& Palla, F. 1997, ApJ, 474, 1

Timmermann, R. 1996, ApJ, 456, 631

Uehara, H., \& Inutsuka, S. 2000, ApJ, 531, L91

van Meersche, M. 1951, Bull. Soc. Chim. Belges, 60, 99

Varandas, A. J. C., Brown, F. B., Mead, C. A., Thrular, D. G., \& Blais, N. C. 1987, J. Chem. Phys., 86, 6258

Watson, W. D. 1976, Rev. Mod. Phys., 48, 513

Watson, W. D., Christensen, R. B., \& Deissler, R. J. 1978, A\&A, 65, 159

Westenberg, A. A., \& de Haas, N. 1967, J. Chem. Phys., 47, 1393

Wolf Savin, D. 2001, ApJ, in press (astro-ph/0109356)

Zhang, J. Z. H., \& Miller, W. H. 1989, J. Chem. Phys., 91, 1528

Zhao, Z. X., Igarashi, A., \& Lin, C. D. 2000, Phys. Rev. A, 62, 042706 
Table 1. Rate COEFficients

\begin{tabular}{llll}
\hline \hline$\#$ & \multicolumn{1}{c}{ reaction } & \multicolumn{1}{c}{ rate coefficient $\left(\mathrm{cm}^{3} \mathrm{~s}^{-1}\right)$} & \multicolumn{1}{c}{ reference } \\
& & & \\
\hline$(1)$ & $\mathrm{D}+\mathrm{H}_{2} \rightarrow \mathrm{HD}+\mathrm{H}$ & $1.69 \times 10^{-10} e^{\left(-4680 / T+198800 / T^{2}\right)}$ & Mielke et al. (1994) \\
$(2)$ & $\mathrm{D}^{+}+\mathrm{H}_{2} \rightarrow \mathrm{HD}+\mathrm{H}^{+}$ & $10^{-9} \times\left[0.417+0.846 \log T-0.137(\log T)^{2}\right]$ & Gerlich (1982) \\
$(3)$ & $\mathrm{HD}+\mathrm{H} \rightarrow \mathrm{D}+\mathrm{H}_{2}$ & $5.25 \times 10^{-11} e^{\left(-4430 / T+173900 / T^{2}\right)}$ & Shavitt (1959) \\
$(4)$ & $\mathrm{HD}+\mathrm{H}^{+} \rightarrow \mathrm{D}^{+}+\mathrm{H}_{2}$ & $1.1 \times 10^{-9} e^{-488 / T}$ & Gerlich (1982) \\
$(5)$ & $\mathrm{H}^{+}+\mathrm{D} \rightarrow \mathrm{H}+\mathrm{D}^{+}$ & $2.00 \times 10^{-10} T^{0.402} e^{-37.1 / T}-3.31 \times 10^{-17} T^{1.48}$ & Wolf Savin (2001) \\
$(6)$ & $\mathrm{H}+\mathrm{D}^{+} \rightarrow \mathrm{H}^{+}+\mathrm{D}$ & $2.06 \times 10^{-10} T^{0.396} e^{-33.0 / T}-2.03 \times 10^{-9} T^{-0.332}$ & Wolf Savin (2001) \\
\hline
\end{tabular}




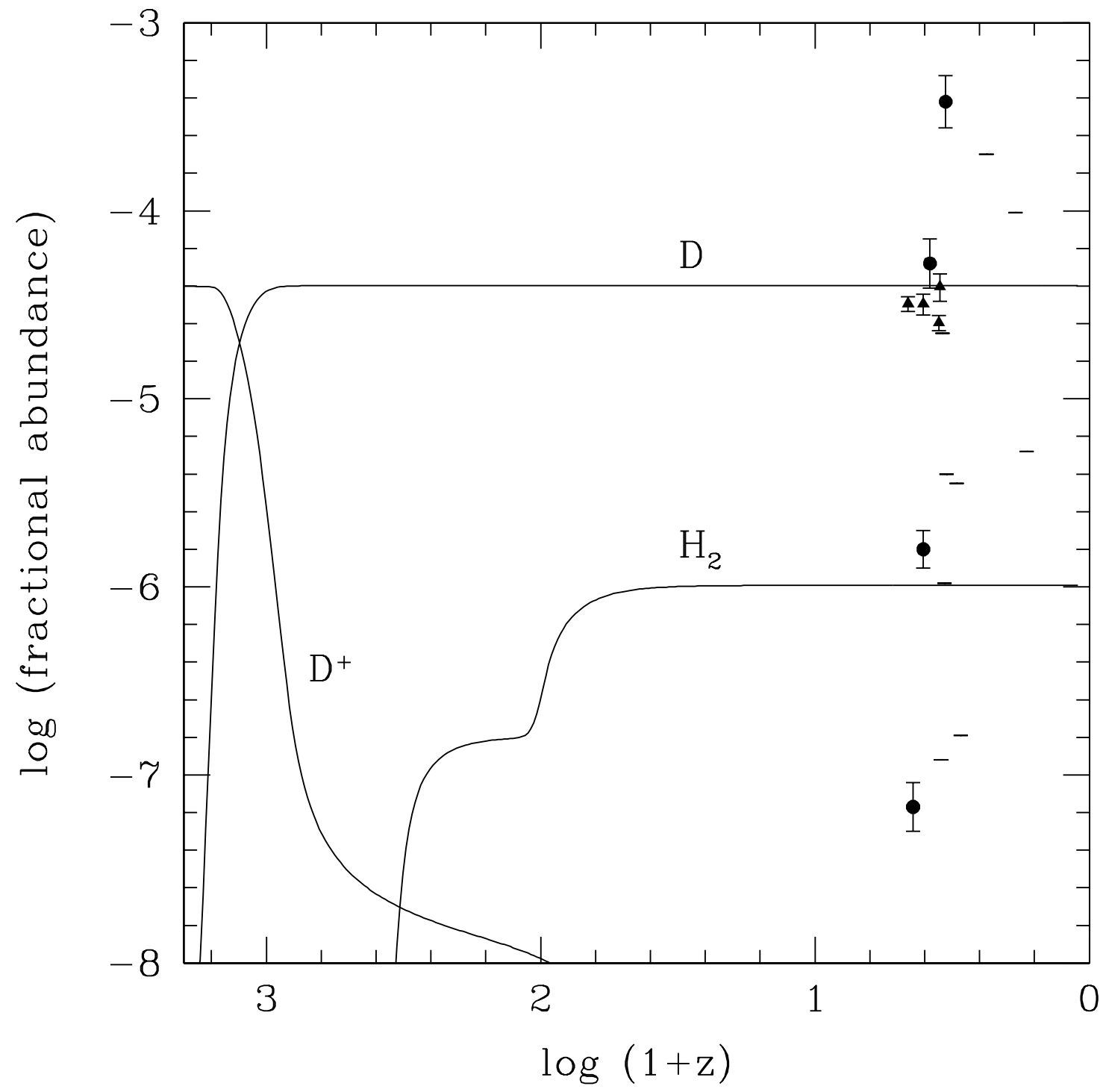

Fig. 1. - Comparison of the abundances of $\mathrm{D}, \mathrm{D}^{+}$and $\mathrm{H}_{2}$ in the primordial gas, relative to hydrogen, as function of redshift $z$ calculated with the standard model of GP. Data points represent abundance measurements of $\mathrm{H}_{2}$ and $\mathrm{D}$ in damped Ly- $\alpha$ systems. The $\mathrm{H}_{2}$ data are taken from the list compiled by Levshakov et al. (2000) (detections, dots with errorbars; dashes: upper limits), whereas D data (triangles with errorbars) are from Burles \& Tytler (1998a,b), O'Meara et al. (2001) and D'Odorico et al. (2001). 


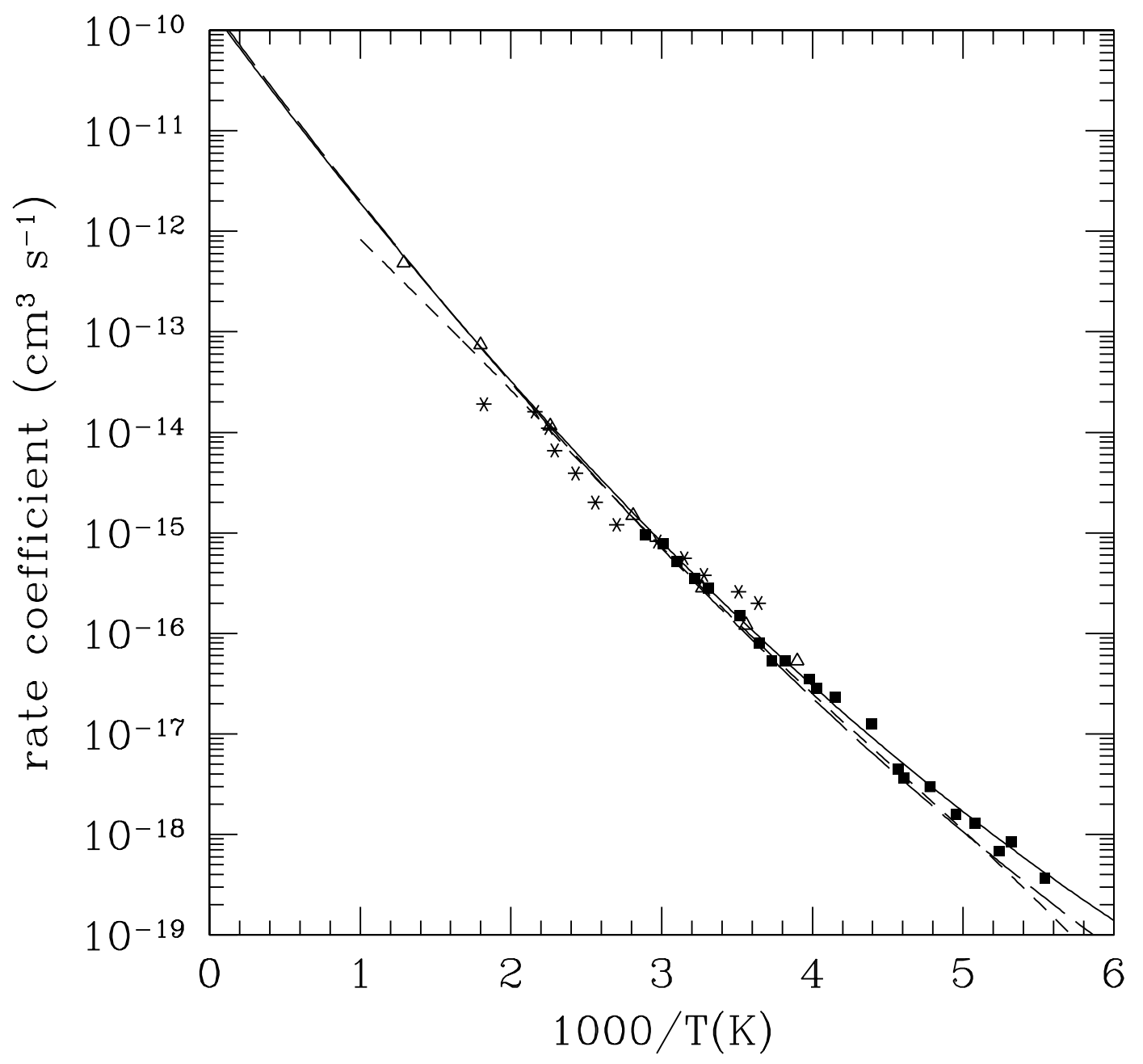

Fig. 2.- Rate coefficient for the reaction $\mathrm{D}+\mathrm{H}_{2} \rightarrow \mathrm{HD}+\mathrm{H}$ according the calculations of Charutz et al. (1997) (short-dashed line), Zhang \& Miller (1989) (long-dashed line), Mielke et al. (1994) (solid line). Experimental data are from Mitchell \& LeRoy (1973) (filled squares), Westenberg \& deHaas (1967) (open triangles), Ridley et al. (1966) (asterisks). 


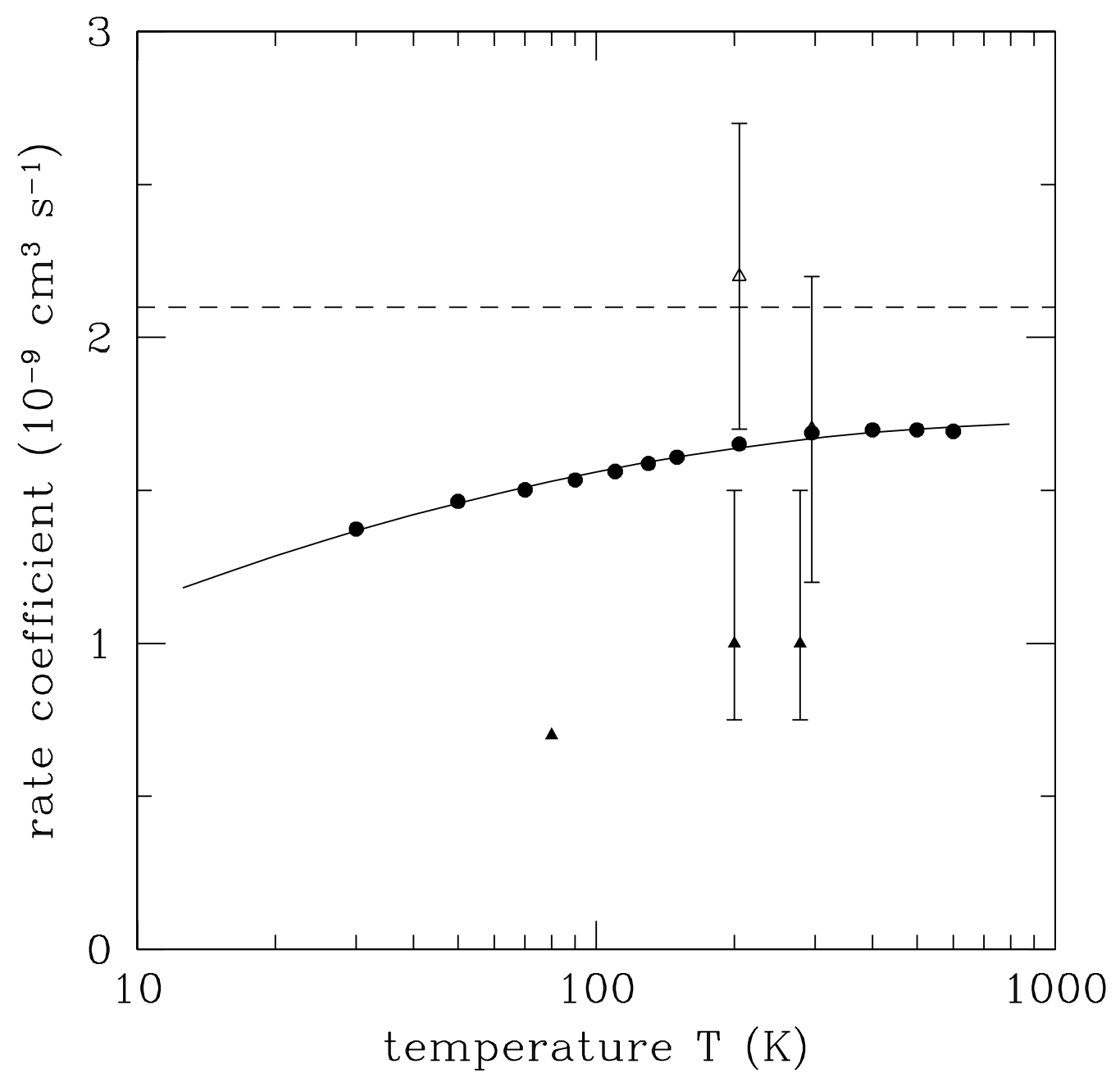

Fig. 3.- Rate coefficient for the reaction $\mathrm{D}^{+}+\mathrm{H}_{2} \rightarrow \mathrm{HD}+\mathrm{H}^{+}$according to the calculations of Gerlich (1982) (filled dots) and the measuruments by Fehsenfeld et al. (1973) (filled triangles) and Henchman et al. (1981) (empty triangles). The dashed line shows the Langevin value of the rate coefficient. The solid line shows our fit to Gerlich's results (see Table 1). 


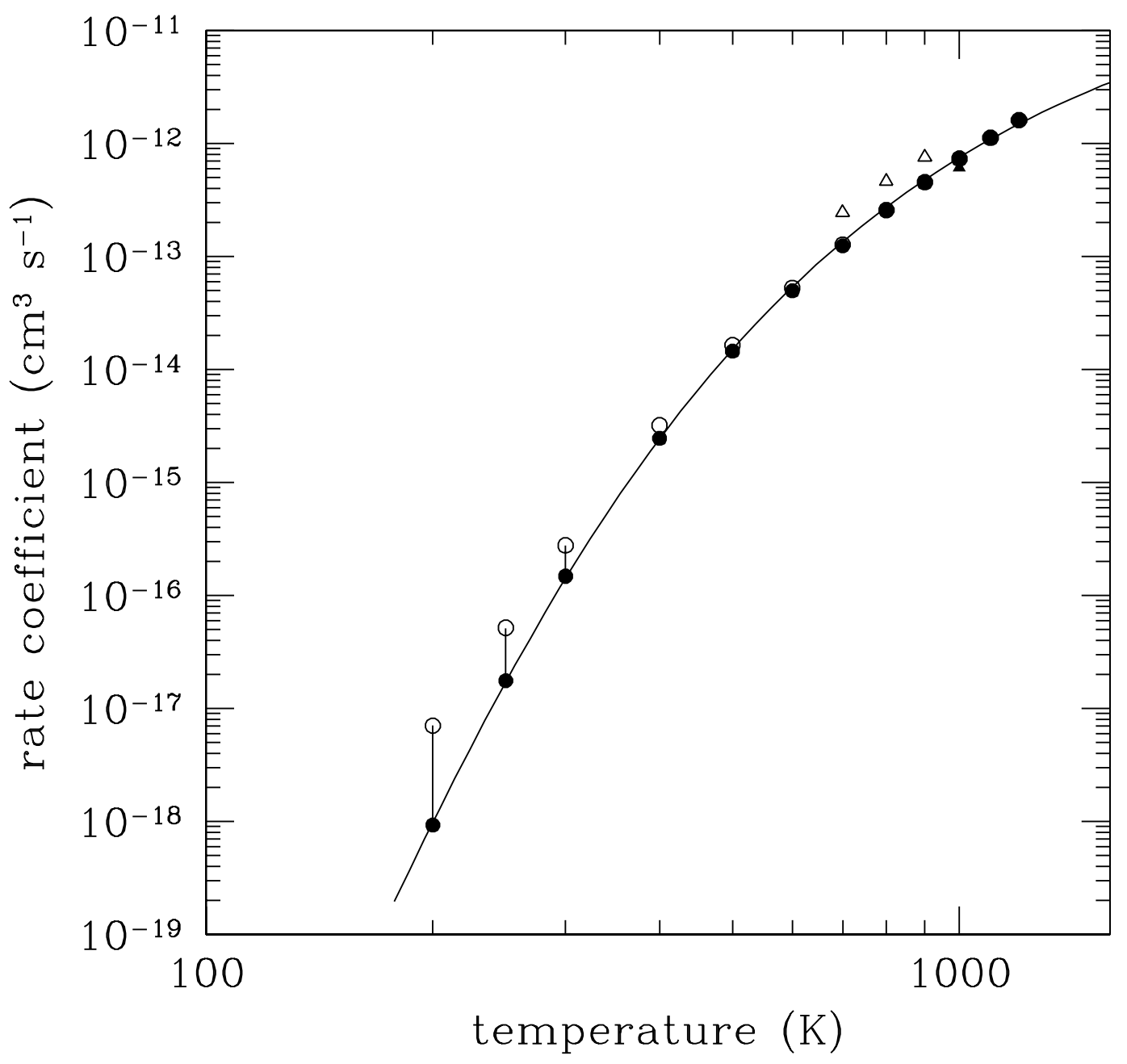

Fig. 4.- Rate coefficient for the reaction $\mathrm{HD}+\mathrm{H} \rightarrow \mathrm{D}+\mathrm{H}_{2}$ according to the calculations of Shavitt (1959) for two values of the asymmetric stretching force constant $A_{u}$ (filled dots: $A_{u}=$

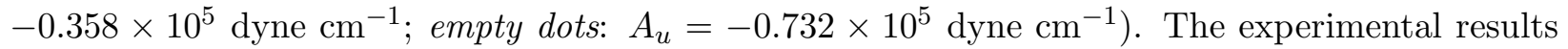
of Boato et al. (1956) and van Meersche (1951) are shown by filled triangles and empty triangles, respectively. The solid line is our fit to the data of Shavitt (1959) for $A_{u}=-0.358 \times 10^{5} \mathrm{dyne} / \mathrm{cm}^{-1}$ (see Table 1). 


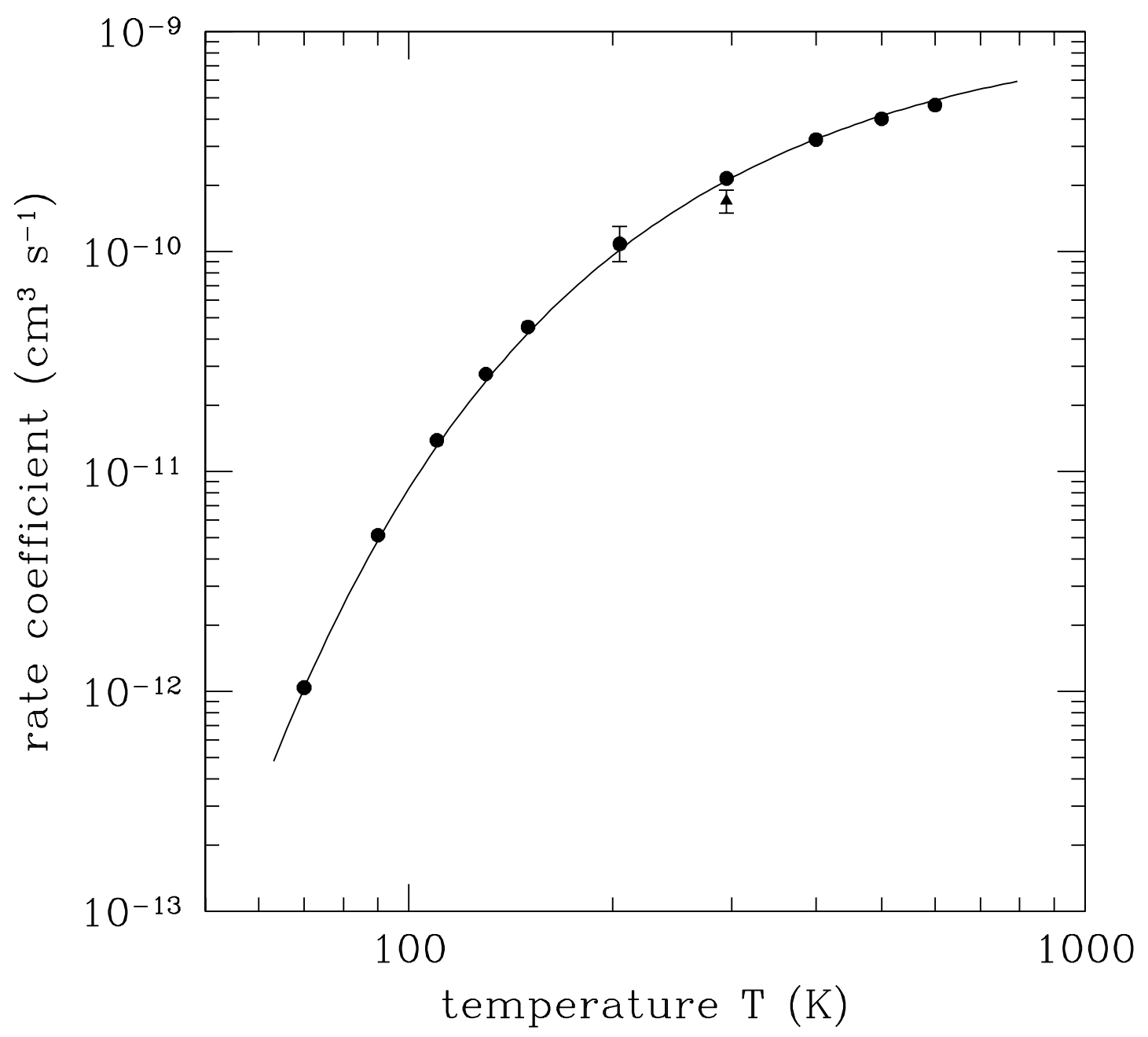

Fig. 5.- Rate coefficient for the reaction $\mathrm{HD}+\mathrm{H}^{+} \rightarrow \mathrm{D}^{+}+\mathrm{H}_{2}$ according to the calculations of Gerlich (1982) (filled dots and the exprimental results of Henchman et al. (1981) at $T=205$ and $295 \mathrm{~K}$ (triangles with errorbars). The solid line shows our fit to Gerlich's results (see Table 1). 


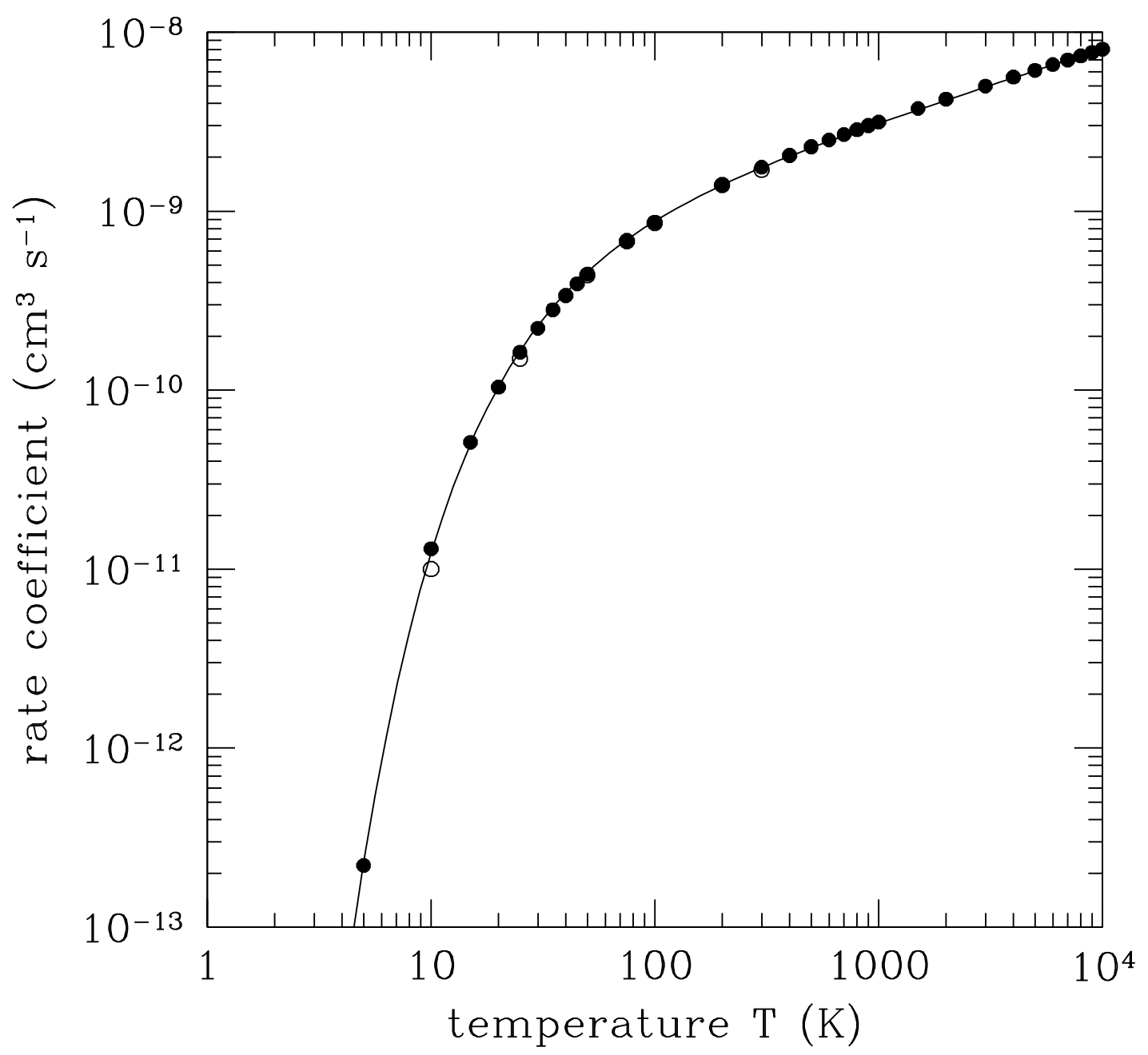

Fig. 6. - Rate coefficient for the reaction $\mathrm{H}^{+}+\mathrm{D} \rightarrow \mathrm{H}+\mathrm{D}^{+}$according to the calculations of Wolf Savin (2001) (filled dots) and Watson et al. (1978) (empty dots). The solid line shows the fit obtained by Wolf Savin (2001) to his results (see Table 1). 


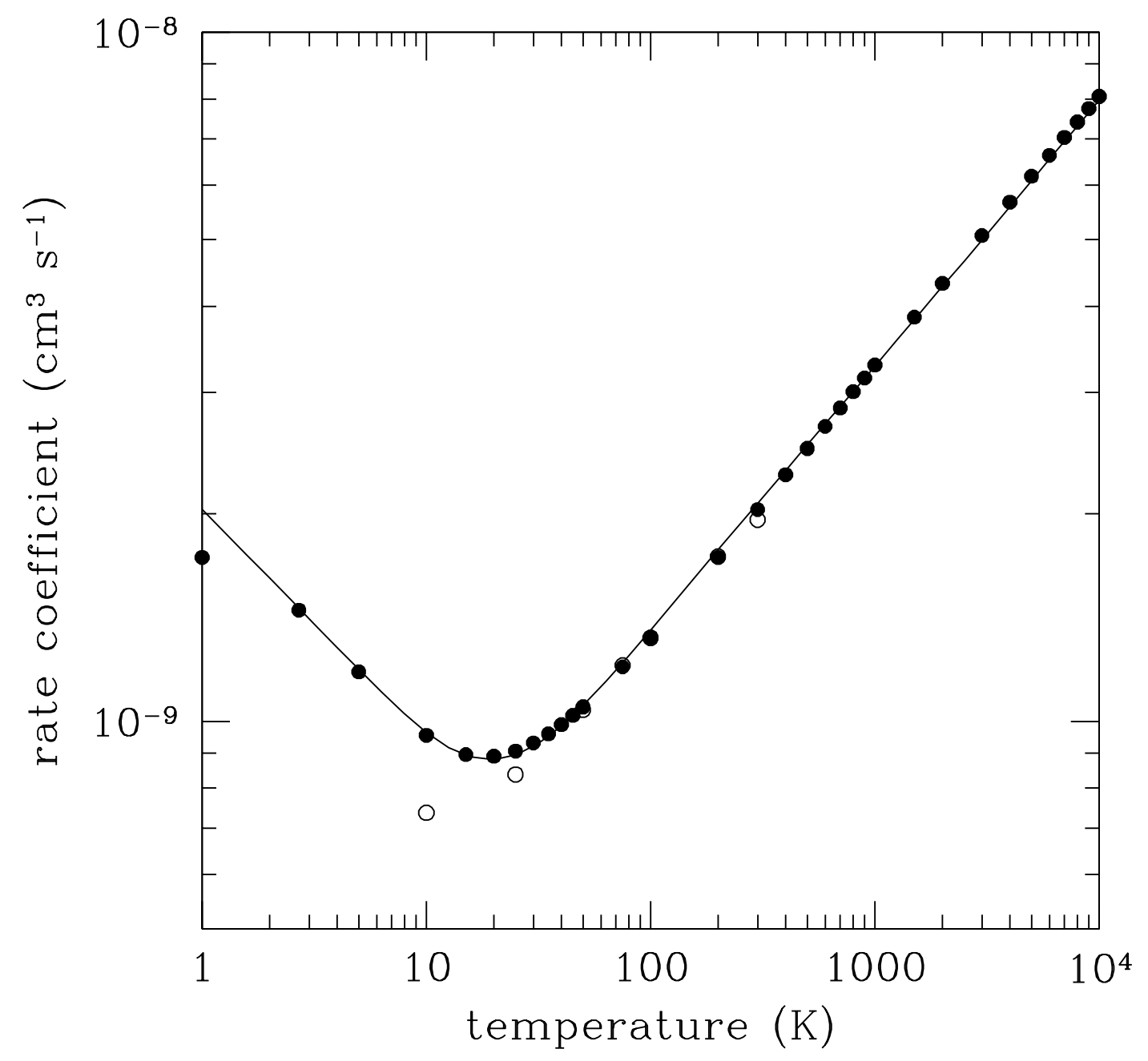

Fig. 7.- Rate coefficient for the reaction $\mathrm{D}^{+}+\mathrm{H} \rightarrow \mathrm{D}+\mathrm{H}^{+}$according to the calculations of Wolf Savin (2001) (filled dots) and Watson et al. (1978) (empty dots). The solid line shows the fit obtained by Wolf Savin (2001) to his results (see Table 1). 


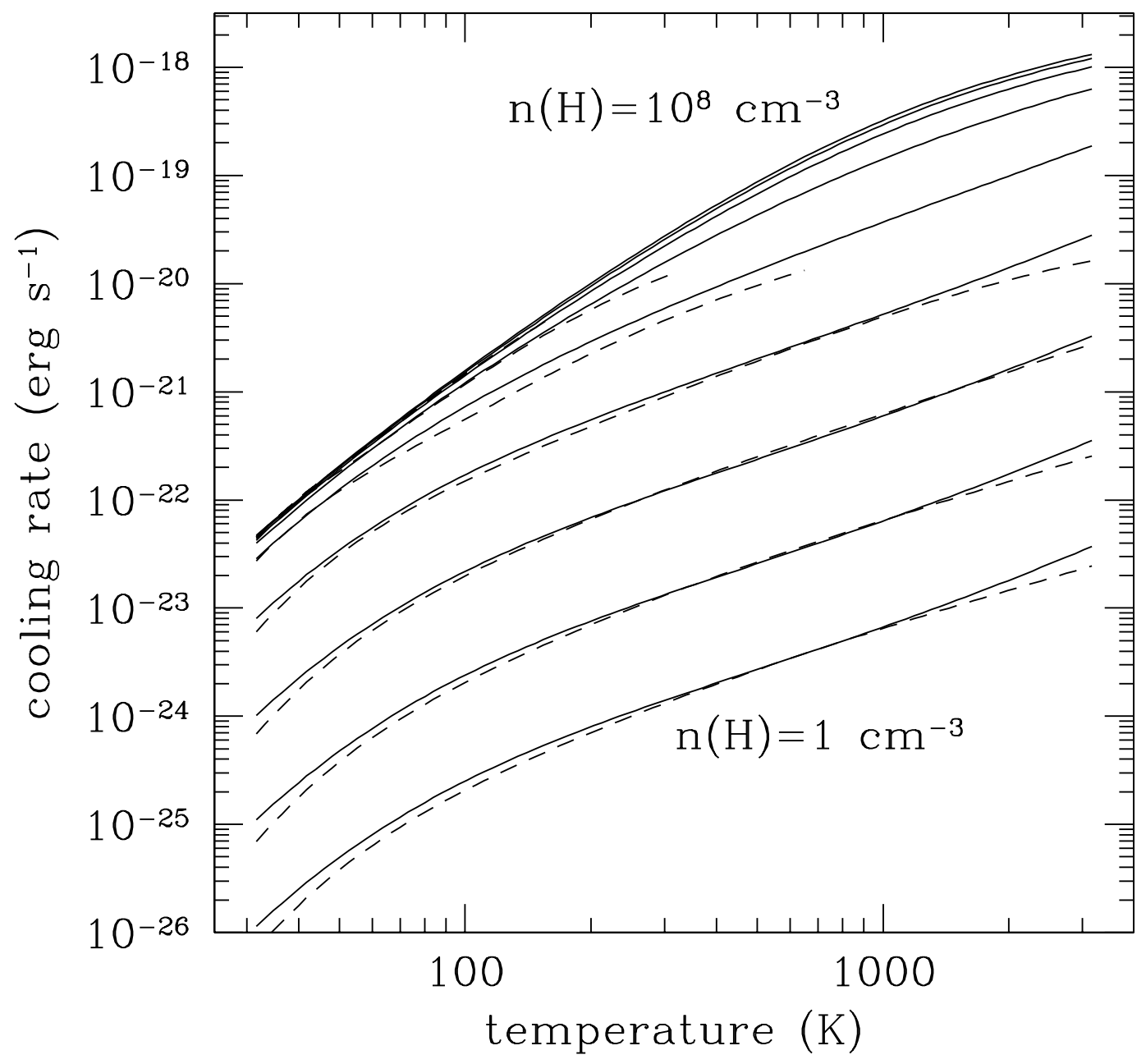

Fig. 8. - The cooling rate per HD molecule computed for $n(\mathrm{H})=1$ to $10^{8} \mathrm{~cm}^{-3}$ (solid lines: Flower et al. 2000; dashed lines: GP). Only collisions of HD with $\mathrm{H}$ have been considered. 


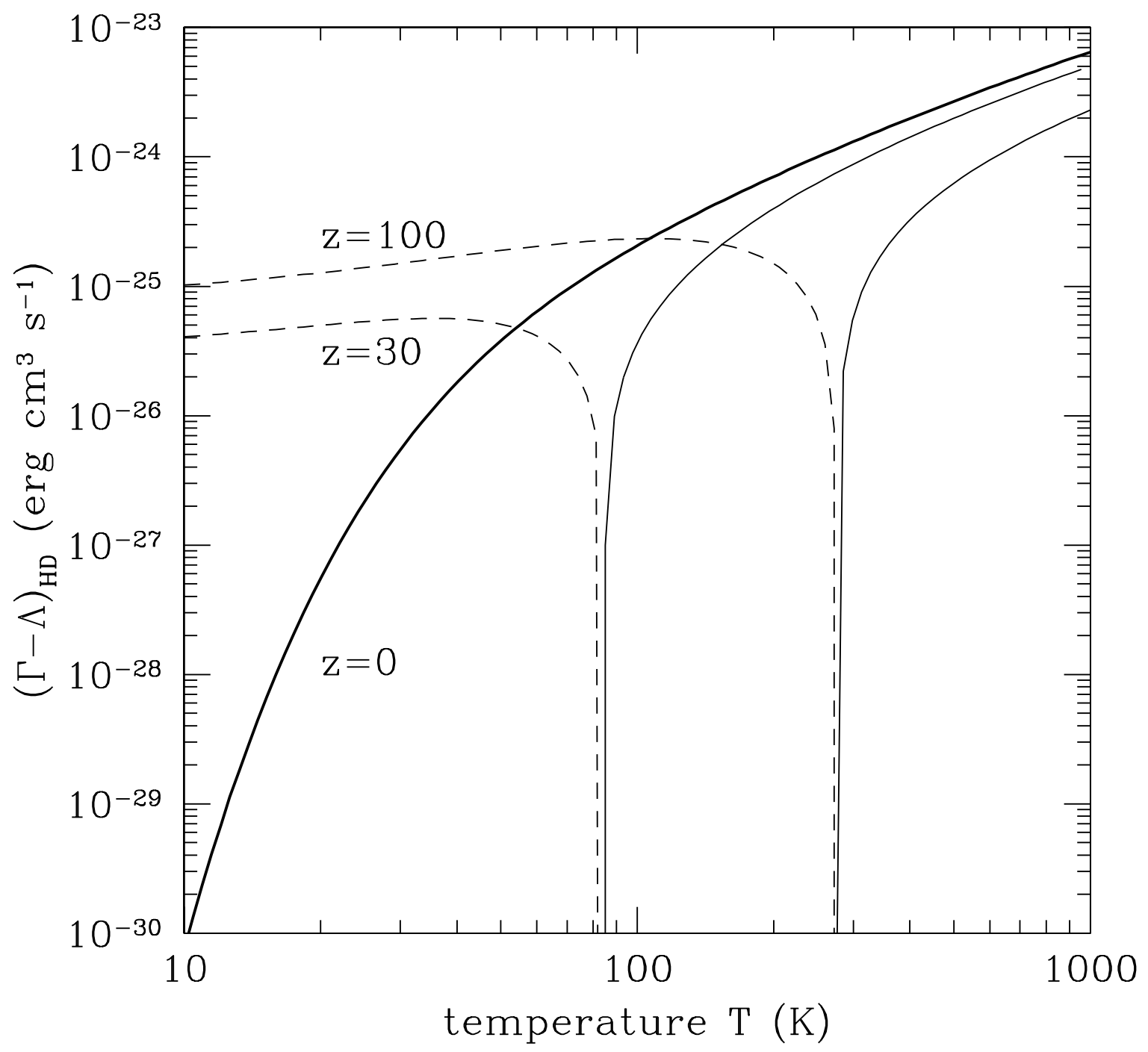

Fig. 9.- The heat transfer function $(\Gamma-\Lambda)_{\mathrm{HD}}$ for $n(\mathrm{H})=1 \mathrm{~cm}^{-3}$ versus gas temperature at selected redshifts. The solid line is computed ignoring the effects of the CBR $(z=0)$. When $T_{\text {gas }}>T_{\text {rad }}$ the heat exchange is a cooling term (solid lines). In the opposite case, the transfer becomes a heating source for the gas (dashed lines). 


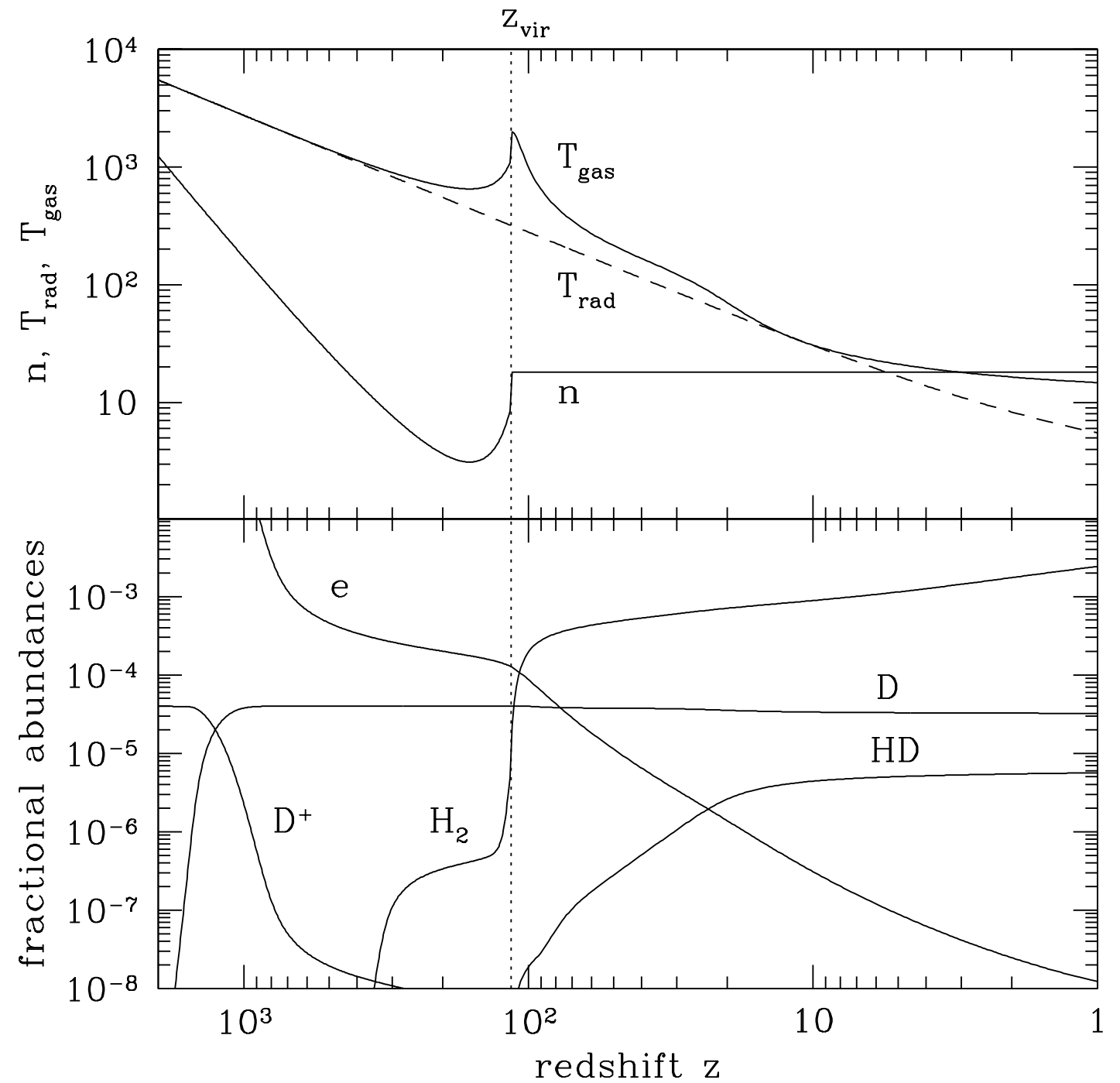

Fig. 10. - The time evolution of gas in a primordial cloud for $z_{\text {vir }}=110, T_{\text {vir }}=2000 \mathrm{~K}, h=0.5$, and $\Omega_{b}=0.06$. The mass of the cloud is $1.35 \times 10^{6} M_{\odot}$. The top panel shows the gas density $n$ (in $\mathrm{cm}^{-3}$ ), the gas temperature $T_{\text {gas }}$ (in $\mathrm{K}$ ) and the temperature of the cosmic background radiation $T_{\text {rad }}\left(\right.$ in $\mathrm{K}$ ) as function of redshift $z$. The bottom panel shows the abundances of electrons, $\mathrm{D}, \mathrm{D}^{+}$, $\mathrm{H}_{2}$ and $\mathrm{HD}$, relative to $\mathrm{H}$, for the same model. 